\title{
EL RETO DE LA INVESTIGACIÓN CUALITATIVA EN PSICOLOGÍA: ALCANCES PARA UN CAMBIO PARADIGMÁTICO EN LA UNIVERSIDAD
}

\author{
The challenge of qualitative research in psychology: scopes for a paradigmatic change in the \\ university
}

\author{
Eric Arenas Sotelo ${ }^{1, \mathrm{a}, ~ \risingdotseq, ~ G u i d o ~ T o r r e s ~}{ }^{1, \mathrm{~b}}$ \\ 1 Universidad Andina del Cusco, Cusco, Perú. \\ a Magíster en Psicología Comunitaria \\ b Doctor en Psicología \\ Đearenas@uandina.edu.pe
}

Citar como: Arenas, E., Torres, G. (2018). El reto de la investigación cualitativa en psicología: alcances para un cambio paradigmático en la universidad. Rev Yachay, 7(1), 484-489.

Recibido: 16-09-2018; Aceptado 07-12-2018

\section{Introducción}

El paradigma cualitativo busca explorar el conjunto de significados, construcciones, percepciones, concepciones, discursos y representaciones sociales e individuales sobre un determinado tema que se pretende investigar. La investigación cualitativa debe dar cuenta de la diversidad de opiniones y creencias dentro de un segmento social, como también debe permitir abstraer conocimiento a nivel teórico. Asimismo, desde la metodología cualitativa se pretende comprender realidades complejas y diversas, considerando las realidades como un sistema que interactúa y se transforma en relación con otros. Además, implica un proceso de construcción teóricopráctica, pues tanto investigador cuanto los participantes, a través de la comunicación e intercambio de ideas, describen y comprenden la realidad para luego ser sustentada en un estudio sistemático y ordenado. Este tipo de investigación tiene como medio y fin la construcción del conocimiento que, además emerge de la subjetividad de la realidad, es decir que la producción de conocimiento sea a través de las personas, las teorías, los tratados y la experiencia de los diferentes actores sociales (Álvarez-Gayou, 2012).

Para de Souza, Ferreira y Gomes (2012) la epistemología cualitativa aborda de forma científica la subjetividad, considerada como un sistema que interacciona y se transforma en relación a otros sistemas- contextos. Desde esta propuesta aparece una crítica al positivismo. La psicología aún queda relegada al dogma de la cientificidad planteado por este paradigma. Asimismo, esta disciplina -como propuesta positivista- cree estar observando y consiguiendo evidencias medibles además de replicables; es decir, como reglas simples, asimiladas por los estudiantes de la psicología sin mayor cuestionamiento teórico ni epistemológico. Sin embargo, esta cientificidad no contribuye al entendimiento del sujeto desde su complejidad. El haber tomado el concepto de medida desde la ingeniería, nos da la respuesta a la asignación de una ciencia positivista. Un positivismo de poca relación con los fenómenos psicológicos, salvo que sean medibles y repetibles que nos habla de validez y confiabilidad para la aprobación de un conocimiento como científico, es decir, la psicología termina convirtiéndose en una ciencia monopolizada entre lo positivista, medible y válido, en mutua relación con el instrumentalismo planteado por dicho modelo -positivista-.

En respuesta a los planteamientos del positivismo en la psicología, la investigación cualitativa propone argumentos que impliquen un debate teórico y metodológico. En ese sentido, busca comprender la complejidad del ser humano -la subjetividad- desde una perspectiva heurística e invención para la producción del conocimiento mediante una metodología asociada al estudio de los procesos psicológicos (Sandoval, 1996).

Zavalloni (en González Rey, 2006) indica que la realidad es un sinfín de campos interrelacionados y que el conocimiento tiene que ser pensado como un proceso constructivo y de interpretación, mas no de medición y término absoluto. El conocimiento y la realidad siempre están en constante construcción y resignificación, denominado por González Rey (2006) como zonas de sentido que, en relación a las ideas de sentido subjetivo, textualmente serían espacios de inteligibilidad producidos en la investigación. Entonces, el pensamiento debería partir desde la creatividad del investigador hasta un sentido especulativo (de curiosidad por saber y conocer) en relación a los 
procesos subjetivos, trascendiendo, de esta manera, a lo empírico. En lo metodológico, se busca legitimizar lo singular para la producción de conocimiento desde ciertas aproximaciones ideográficas pues el positivismo buscará una proposición de ley donde el individuo es la sumatoria de una estadística más.

Se propone un método constructivo-interpretativo pues el conocimiento es de carácter interpretativo que va más allá de solo superar la validez y confiabilidad de una construcción simplificada y arbitraria de la realidad. La construcción del conocimiento va en relación a la producción de zonas de sentido. La epistemología cualitativa debe ser entendida como un proceso de comunicación, diálogo -como ontología humana- e interpretación mutua del espacio social y la resignificación de la información producida a través del uso permanente de la comunicación y el lenguaje (Lizcano, 2013).

Un modelo cuantitativo definido desde la perspectiva positivista en las ciencias sociales y de la salud, será aquel que solo enfatiza lo empírico y descriptivo de la investigación. De acuerdo a esto, la investigación se convierte en un ejercicio instrumentalista y ateórico, de un recojo acumulativo de datos cuantificables y con verificación inmediata, de evidencias observables apoyadas en las estadísticas. En ese sentido, prepondera la hegemonía del instrumentalismo, la cuantificación en su sentido más amplio y dominante en el que los instrumentos y técnicas se imponen a epistemologías teóricas de reflexión sobre la realidad, además de la aglomeración de información y clasificación de un conjunto de conocimientos existentes, mas no reinterpretados o producidos significativamente (Flick, 2014). En esa línea, la ignorancia del positivismo sobre cuestiones importantes en la generación del conocimiento deja grandes vacíos al no querer entender la realidad más allá de los números y la propuesta naturalista.

Ahora bien, la respuesta desde el paradigma cualitativo crítico, va desde el significado esencial de una producción teórica con ideas y modelos de interpretación y reflexión. La postura de la epistemología cualitativa invita a pensar en una apuesta de construcción productiva del conocimiento y un método relacionado a la dialogicidad. Este último es planteando como un proceso de comunicación y como tal será una vía para el conocimiento de lo subjetivo del individuo y la sociedad, de los espacios donde se desenvuelven que, de hecho, también son interpretaciones de los sentidos subjetivos.

\section{Desarrollo}

La realidad y la construcción del conocimiento muestra una gran variedad e inmensas posibilidades de explorar, describir y comprender los distintos fenómenos psicológicos, sociales e intersubjetivos (Álvarez-Gayou, 2012). Existe una pluralidad dentro de la vida social del ser humano y esto hace que los distintos procesos psicosociales dentro de la ciencia, también sean heterogéneos. En ese sentido, se pueden encontrar varios cambios que se reflejan en la sociedad y, por tanto, el conocimiento no está lejos de la mirada democrática diversa de la producción y creación de los saberes.

La psicología en su mirada clásica de la ciencia frente a una nueva propuesta epistemológica a un inicio -en el proceso de validarse como ciencia desde las propuestas del positivismo- atendía los asuntos de la ciencia desde lo psicofísico, enmarcada en la lógica del método experimental. Tradicionalmente la psicología se basó temas estandarizados: con parámetros de frecuencia y distribución numérica (Flick, 2007). En cambio, actualmente la psicología se mueve en dos dimensiones: las neurociencias y lo psicosocial; es decir, la persona como sujeto de estudio en la disciplina neurocientífica, y el contexto desde una mirada psicosocial y sociocultural. Asimismo, también se puede plantear una perspectiva filosófica y epistemológica de esta disciplina desde un panorama cualitativo, aunque todavía se dé como sustento de esta, el positivismo y su modelo hegemónico. Según Sánchez (2012) se dan un par de supuestos importantes para explicar este modelo: 1) la realidad y la objetividad que determina que el conocimiento es independiente del investigado, y 2) la causalidad, que implica una causa eficiente del efecto. En respuesta a esto, se plantean modelos alternos que explican la ciencia en relación a los procesos metodológicos y las reflexiones epistemológicas para la generación de conocimiento no son solo unidimensionales; puesto que pueden existir otros caminos para la producción del conocimiento, que también son sistemáticos y organizados. De esta manera, se puede establecer el diálogo de la psicología con otras ciencias y disciplinas que permitan presentar nuevas formas en la generación del conocimiento.

La investigación cualitativa desde y para la psicología, busca conocer la realidad -la percepción y conocimiento de esta- como una construcción y resultado de redes de conocimiento, sean de acción social o por medio del lenguaje y sus códigos interpretativos. Por eso, la realidad debe ser entendida como un todo, como un conjunto holístico que se pueda interpretar de manera integral en el contexto de su producción (Sánchez, 2012). Es por eso, que se reconoce el rol trascendente del investigador a la hora de generar conocimiento, quien asume un papel reflexivo y crítico e interactúa con los participantes, situación que difiere con la supuesta neutralidad que se plantea en el otro panorama teórico.

En la comunidad científica de la psicología, se debe entender de manera general, que la investigación cualitativa ha quedado postergada por dificultades como la validación del conocimiento generado desde esta disciplina, el poco interés por hacer estudios de esta naturaleza y el mínimo financiamiento para desarrollar investigaciones. Situaciones que también han generado la invisibilización de esta propuesta teórica de generación de conocimiento. La psicología cualitativa busca generar una psicología que construya la realidad junto al otro el sujeto- y este como una fuente de provisión de datos e información. Ibáñez (2001) indica que hay algunos mitos que la psicología 
debe ir superando para pensar en una psicología desde lo cualitativo. Estos son: a) el mito de la representación en la cual la realidad es independiente de los eventos y del conocimiento, b) el mito del objeto donde la realidad debe ser neutral al investigador, c) el mito de la independencia de la realidad-en la cual la realidad debe estar en función de los criterios que tenemos para explicarla, y d) el mito de la verdad- en la cual la verdad es fáctica frente a las verdades particulares construidas y contingentes al sujeto - .

Ahora bien, ambos enfoques cuantitativo y cualitativo de estudio de la realidad tienen sus bondades y más que antagónicos, pueden ser complementarios, es decir, pueden encontrar puentes en los que converjan una línea argumentativa que permita plantear acciones de cambio en la sociedad desde la psicología. Sin embargo, existen diferencias sustantivas entre ambas que deben de ser distinguidas. Por ejemplo, pedirle a un pez que vuele, sabiendo que su habitad natural es el agua. En la línea del ejemplo, sería como ir contra la naturaleza misma de cada paradigma de investigación si juzgamos a uno con las reglas del otro. Eso quiere decir que, cada enfoque tiene sus normas y fundamentos teórico- metodológicos que respaldan sus planteamientos y es, a partir de estos, cómo deben ser sometidos a la opinión de la comunidad científica. En ese sentido, ambos distinguen diferentes valores y formas de concebir la realidad. Cabe mencionar que el enfoque cualitativo se da a partir del reconocimiento que se puede ir generando conocimiento respecto a: significaciones, pluralidad, interpretaciones y el rol clave del sujeto como productor de saberes. Que, además, implica también una crítica y reflexión desde ambas posturas de concepción de la realidad, a partir de las diferencias y aportes que puedan brindar a las disciplinas científicas.

Una de las cuestiones que hasta ahora se encuentra en discusión, es saber a qué clasificación de las ciencias pertenece la psicología, es decir, en qué disciplina está enfilada y cómo fundamenta sus planteamientos teóricos. Se ha señalado que podría estar en las ciencias de la salud como también en las ciencias de las humanidades; podría también encontrarse entre las ciencias sociales (González, 2003). Los paradigmas son universalmente y reconocidos en la comunidad científica; es decir, el paradigma respalda y diferencia a una comunidad científica de otra. También, los paradigmas responden a principios y supuestos básicos como: supuesto ontológico (naturaleza de la realidad), supuesto epistemológico (naturaleza del conocimiento), supuesto metodológico (medio para obtener conocimiento). Respecto al paradigma positivista, se distingue la realidad que se estudia con los siguientes planteamientos: 1) señala que existe solo una realidad, 2) el mundo es un "uno" independiente de cómo se pueda estudiar, y 3) se rige por leyes que explican, predicen y controlan los fenómenos estudiados. Entonces, para el positivismo, la ciencia tiene que generar leyes, hacer generalizaciones y promover un conocimiento universal (Lizcano, 2013).

Entendiendo a la psicología como una ciencia que pertenece a las ciencias sociales, pretender poner sus planteamientos como válidos desde el positivismo es una limitante. En esa línea, para la psicología entendida desde lo cualitativo, las realidades son particulares y se pueden estudiar desde las ciencias sociales de manera compleja y completa. La propuesta cuantitativa plantea que, para hacer confiable un estudio, se necesita validar por criterios estadísticos, sea en la muestra o el instrumento; condición que además validará los resultados de manera universal en diferentes contextos y situaciones. Sin embargo, estos supuestos se acercan más a las ciencias naturales que a las sociales. Mardones y Ursúa (1982) indican las siguientes características de esta propuesta: el monismo metodológico, el modelo o canon de las ciencias naturales exactas, la explicación causal para la explicación científica y la búsqueda de un interés dominador.

Tenemos al paradigma interpretativo, en el cual la perspectiva cualitativa se acerca más a los estudios desde las ciencias sociales. Es decir, esta propuesta se acerca con mayor propiedad al estudio de fenómenos de carácter social, conociendo, de esta manera, particularidades como: lo individual, lo distintivo, las realidades múltiples y lo particular de un hecho (Pedraz, Zarco, Ramasco y Palmar, 2014). Además, los postulados de una teoría sirven para espacios y tiempos determinados y también pueden generar teorías con intención de explicar otras realidades. Asimismo, se busca descubrir el significado de las acciones humanas, la cuestión social, el mundo personal, motivaciones y creencias de un grupo social o un individuo. Lo cualitativo sigue una lógica inductiva, por eso se construyen categorías, comprensiones e interpretaciones que parten de datos obtenidos en el proceso de investigación (Lizcano, 2013). Existe una mutua interacción entre el investigador y lo investigado. Este proceso se da de forma circular y puede reformularse constantemente. Así también se encuentran algunos enfoques cualitativos como la fenomenología, la hermenéutica, etnografía, interaccionismo simbólico y el construccionismo social, entre otros, que permiten conocer la realidad desde los planteamientos particulares y diversos que proponen. Este paradigma tiene las siguientes características (Patton citado por Merino, 1995): un tipo de análisis inductivo como un proceso holístico que brinda datos cualitativos, además se da un contacto personal, genera sistemas dinámicos y se orienta hacia un caso único, contextualiza, mediante una postura de neutralidad empática y flexibilidad metodológica.

Otra propuesta teórica es el paradigma sociocrítico que reacciona -responde- a los anteriores paradigmas; al positivismo por su reduccionismo, objetivismo y lo conservador (Mardones y Ursúa, 1982) y al interpretativo por su tendencia al subjetivismo. Esta propuesta paradigmática, genera una conciencia emancipadora y entiende a la ciencia y el conocimiento como un camino para la liberación del hombre, la investigación como una propuesta trasformadora y de construcción social de la realidad (Gergen, 2007). Entonces este paradigma propone: una concepción social, pluralista e igualitaria de la realidad y la producción de conocimiento. Por eso, el ser humano es coautor de la realidad; y la realidad es resultado de las prácticas humanas (Luckmann y Berger, 1968). Los enfoques investigativos que se proponen son: la investigación acción, investigación colaborativa y la investigación participativa, como 
también la investigación acción participativa. Por eso se busca generar tres procesos: acción, práctica y cambio. En razón de cada uno de los planeamientos de esta propuesta, se fundamenta y se usa el conocimiento en y para la acción trasformativa.

\section{Análisis y discusión}

Las tensiones y acercamientos entre los paradigmas durante el proceso de investigación, implican una suerte de distanciamientos teóricos y pragmáticos. Asumir uno u otro paradigma depende de la cosmovisión del investigador, sus presupuestos teóricos y metodológicos; por eso, resulta difícil la fusión de ambos paradigmas (aunque podría existir una complementariedad entre ambos). Sin embargo, no se debe olvidar que toda propuesta metodológica corresponde al paradigma de investigación o a la combinación de ambos.

La investigación como propuesta cualitativa implica planteamientos de una psicología socio-crítica que, además, busca aportar a la sociedad mediante la producción del conocimiento y el entendimiento complejo de las realidades sociales, psicológicas, culturales y comunitarias. El estudio, desde el panorama cualitativo, puede generar conocimiento en todo ámbito de la psicología y como propuesta teórica, son claves los estudios en temas de democracia, ciudadanía, inclusión, cultura, educación, salud y políticas públicas, entre otros. En ese sentido, toda realidad que implique percepciones o significados, podrá ser sometida al análisis interpretativo, cualitativo y social crítico del investigador. El investigador entra en intimidad con el escenario y fenómeno que estudia, ello le permite identificar el contexto, los actores- participantes, las normas socioculturales, los valores, los lenguajes y códigos, como también las jerarquías y estructuras de poder (González, 2003). Asimismo, puede complejizar las interacciones grupales, permite mapear la geografía humana y física de manera temporal y espacial. Lo cualitativo comprende la horizontalidad, el diálogo, los significados y la subjetividad durante el proceso de investigación (Souza, Ferreira \& Gomes, 2012). Por lo general en psicología se busca generar datos supuestamente sólidos y generalizables, brindando una explicación de manera numérica y descriptiva desde los estudios cuantitativos. Sin embargo, desde lo cualitativo, se busca entender la construcción humana de los saberes, el reconocimiento de la subjetividad, la historia y el contexto de los procesos psicológicos desde una lógica interactiva y relacional. La propuesta cualitativa permite comprender la realidad como un todo complejo.

La subjetividad es el objetivo de estudio de la investigación cualitativa. Según Sandoval (1996) el estudio cualitativo busca recuperar la subjetividad como construcción humana, comprender la vida cotidiana de la realidad sociocultural y la intersubjetividad para acceder a la realidad humana. La investigación cualitativa ayuda a comprender procesos simbólicos y subjetivos en una manera de construir identidad y de significar los espacios y procesos sociales (Flick, 2014). El investigador puede establecer un diálogo que permita surgir una verdad que está dada por una participación conjunta y contextualizada. Para González Rey (2006) el sentido subjetivo expresa las producciones simbólicas y emocionales de manera histórica y social de lo que hace el humano.

Entonces, cuando buscamos entender la realidad como un proceso de subjetivación simbólica, la investigación cualitativa se hace clave, pues para conocer realidades relativas y reflexivas, entenderla implica generar conocimiento, además que se pueda buscar un metapunto para reflexionar y relativizar los conocimientos (Peréz, 2012). En ese sentido, la subjetividad social y la subjetividad individual se interrelacionan en una unidad. La subjetividad social como la individual puede verse afectada debido a las condiciones en las que se desenvuelve el sujeto, a su contexto cultural y social histórico.

La realidad psicológica de los sujetos puede ser entendida desde la investigación cualitativa como un proceso complejo desde la psique, la mente y la conciencia; busca la comprensión amplia de estos procesos de construcción de la realidad simbólica y abstracta, concreta y realista. Además, esta realidad puede ser vista por distintos medios o metodologías que permitan usar ciertos instrumentos como la entrevista (Morga, 2012), y la observación para conocer la realidad subjetiva del ser humano. Como también técnicas colectivas como los grupos focales (Montero, 2009) y los grupos de discusión (Peinado, Martin, Corredera, Moñino y Prieto, 2010). Para elegir la muestra se permite desde un criterio intencional y su selección se da por amplitud, variedad e integración de realidades divergentes.

De lo ya revisado hasta este punto, queda la siguiente interrogante ¿es la investigación cualitativa una revolución científica? Los científicos pretenden defender la idea que saber cómo es el mundo. Por eso, la ciencia "normal" a menudo intentará suprimir las novedades que amenazan sus fundamentos. A estos giros- epistemológicos y ontológicos- de la ciencia en un entendimiento heterogéneo y diverso, Kuhn los denomina como revoluciones científicas (Flick, 2007). Entonces, es el arte -de la investigación- que, en su libertad, se convierte en un camino a la creación válida del conocimiento frente a la ciencia. En este caso, el paradigma cualitativo se muestra como un reto, un camino distinto, emancipador y como alternativa a la clásica psicología positivista. Es decir, plantea un conjunto de bases teóricas y metodológicas que permitirán la construcción del conocimiento desde otra arista: una que implique propuestas sociales, críticas y propositivas para generar nuevos saberes y el acercamiento a la realidad. Los estudios en la disciplina psicológica no pueden reducirse a ecuaciones estadísticas o al advenimiento de un test psicológico como punto de partida de una investigación, sino más bien, deben de partir de la curiosidad genuina del investigador para estudiar un problema que realmente quiere conocer de la realidad.

En la cotidianidad se suele usar lo subjetivo para desvalorizar las opiniones del otro o, en todo caso, invalidar las distintas ideas o significados que propone un estudio. Sin embargo, no es la subjetividad en sí misma la que tiene que ser invalidada, pues es esta, el reflejo de lo que cada sujeto o comunidad mantiene y sustente como un conocimiento real y sostenido de los contextos que el

Rev. Yachay volumen (7) Número (1), enero-diciembre 2018 
investigador quiere comprender y estudiar. Y cómo se dará la producción de conocimiento, pues será mediante una reflexión epistemológica y teórica, que permita una psicología de creación del conocimiento (Peréz, 2012).

En esa línea, se debe cuestionar la fuerza de los términos y conceptos en relación a un modelo todavía dominante, asumir una producción teórica e interpretativa de la realidad. Para la producción de conocimiento, se puede discrepar con ese modelo, que entiende que los procesos psicológicos solo pueden ser estudiados desde lo cuantificable, y además, tiene como esencial procedimiento el recojo de información estandarizada, la validez estadística y una sumatoria de evidencias empíricas. Además, este modelo positivista termina siendo limitado pues la producción de conocimiento no tendría que ser el mero hecho de sumar opiniones y por ende sujetos. Es por eso que, la producción de saberes no puede afirmarse como concluido, terminado e inminentemente un conocimiento final; porque a medida que vamos interaccionando con el sujeto y la realidad, el conocimiento también sigue modificándose (González, 2003).

El respaldo teórico para la producción de conocimiento se hace imprescindible en tiempos donde solo lo cuantificable, numerable y estadísticamente comprobable, es válido. Además, el investigador cree que los estudios pueden ser inciertos sin números y mediciones. Contrario a esto, la epistemología cualitativa brinda una alternativa de discusión sobre la realidad y construcción de conocimiento, pues este va más allá de lo instrumental y estadístico. Es decir, el conocimiento merece una discusión teórica y se permite el uso de una metodología que no tema a especular, a darse idea y abstraer los procesos subjetivos, y que permitan respaldar el potencial creativo del investigador (Flick, 2014).

\section{Conclusiones}

Primera. La investigación cualitativa permite entender las diversas realidades y situar el conocimiento de acuerdo al contexto en el que cual se busca comprender un fenómeno de estudio.

Segunda. La investigación cualitativa en psicología implica un proceso de construcción teórica y práctica de conocimiento con fundamentos epistemológicos y ontológicos, pues tanto investigador como participantes, a través de la comunicación e intercambio de ideas, describen y comprenden la realidad.

Tercera. El enfoque cualitativo no está interesado en la explicación de los fenómenos sino en la comprensión de los mismos, es decir, la producción teórica va de la mano con la reflexión del sujeto e investigador. Está relacionado como un proceso subjetivo, que viene de la mano de su historia, creencias, representaciones, valores y otros aspectos que manifiesten su subjetividad.

Cuarta. El conocimiento per se, es un proceso de producción continuo entre la permanente representación/reinterpretación de la realidad y el sujeto en relación a una epistemología teórica que permita sustentar el conocimiento desde una aproximación metodológica más flexible, pero también rigurosa y ordenada.

Quinta. Lo singular toma trascendencia cuando se vale de un modelo teórico para la interpretación de la realidad, ahí toma fuerza la necesidad de plantear un modelo epistemológico, y no solo un uso instrumental de la psicología. Además, la singularidad también está marcada por el espacio social y cultural en el que se encuentra el sujeto como unidad colectiva, como comunidad.

\section{Referencias bibliográficas}

Álvarez-Gayou, J. (2012). Cómo hacer investigación cualitativa: Fundamentos y metodología. México D.F: Paidós.

Flick, U. (2014). La gestión de la calidad en investigación cualitativa. Madrid: Ediciones Morata.

Flick, U. (2007). Introducción a la investigación cualitativa. Madrid: Ediciones Morata.

Gergen, K. (2007). Construccionismo social: Aportes para el debate y la práctica. Bogotá: Universidad de los Andes, Facultad de Ciencias Sociales, Departamento de Psicología, CESO, Ediciones Uniandes.

González, A. (2003). Los paradigmas de investigación en las ciencias sociales. Islas, 45(138), 125-136.

González Rey, F. (2006). Investigación Cualitativa y Subjetividad. Guatemala: Oficina de Derechos Humanos del Arzobispado de Guatemala.

Ibáñez, T. (2001). La psicología social cons-truccionista. Guadalajara, México: Universidad de Guadalajara.

Mardones, J. \& Ursúa, N. (1982). Filosofía de las ciencias humanas y sociales: nota histórica de una polémica incesante, en Filosofía de las ciencias humanas y sociales (materiales para una fundación científica). Fontamara. Barcelona.

Merino, C. (1995). Metodología cualitativa de la investigación psicosocial. UNAM-CISE, España.

Montero, M. (2009). Grupos Focales. Caracas: AVEPSO.

Morga, L. (2012). Teoría y técnica de la entrevista. México D.F: Red Tercer Milenio.

Lizcano, J. (2013). Investigación cualitativa de segundo orden y la comprensión de la realidad. Hallazgos, 10(19), $149-162$.

Luckmann, T., \& Berger, P. (1968). La construcción social de la realidad. Buenos Aires: Amorrortu.

Pedraz, A., Zarco, J., Ramasco, M., \& Palmar, A. (2014). Investigación Cualitativa. Barcelona: Elsevier. 
Peinado, Y., Martin, T., Corredera, E., Moñino, N., \& Prieto, L. (2010). Grupos de discusión: Métodos de investigación en Educación Especial. Madrid: Universidad Complutense de Madrid.

Peréz, A. (2012). Sobre el constructivismo: Construcción social de lo real y práctica investigativa. Revista Latinoamericana de Metodología de las Ciencias Sociales, 2(2), 5-21.

Sánchez, E. (2012). La investigación cualitativa en psicología: ¿Por qué ésta metodología? 83-92.

Sandoval, C. (1996). La formulación y el diseño de los procesos de investigación social cualitativos. Instituto colombiano para el fomento de la Educación Superior. 111-128.

Souza, M., Ferreira, S., \& Gomes, R. (2012). Investigación social: Teoría, método y creatividad. Buenos Aires: Lugar Editorial.
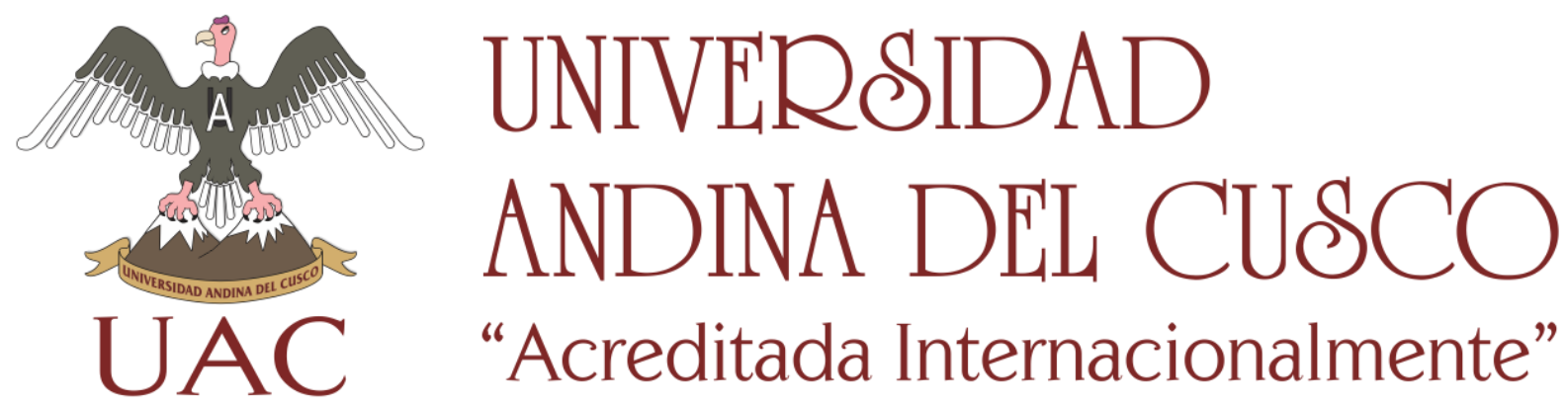